\title{
IMPROVED MODAL CONTRIBUTION FACTORS AS RESPONSE TRACKING MECHANISMS FOR DYNAMIC SYSTEMS DURING DESIGN OPTIMIZATION
}

\author{
Michelle Guzman Nieto*, Mostafa S.A. ElSayed ${ }^{* *}$ \\ Dept. of Mechanical and Aerospace Engineering \\ Carleton University \\ Ottawa, Ontario, Canada \\ *Michelle.guzman@carleton.ca \\ *** Mostafa.elsayed@carleton.ca
}

\author{
Denis Walch \\ Bombardier Aerospace \\ St-Laurent, Quebec, Canada \\ Denis.Walch@aero.bombardier.com
}

\begin{abstract}
As an industrial practice, complex structures are analyzed under a large set of dynamic operating conditions from which design load cases are selected. During design optimization, response tracking mechanisms based on modal participation factors could lead to large computational savings. In this paper we review the use of static modal participation factors as an inexpensive method to approximate the modal response of a dynamic system and extend the analysis to estimate the peak modal response when the structure is subject to a shock or pulse disturbances. A case study is presented as a proof of concept where the derived approximate peak loads are also estimated.
\end{abstract}

Keywords: modal contribution factor, modal contribution factors, dynamic analysis, static modal decomposition

\section{INTRODUCTION}

In practice, complex structures must be analyzed under a large set of dynamic operating conditions to ensure that the design will meet prescribed requirement.

During optimization, a relatively small number of such operating conditions are selected as critical and are used to minimize or maximize the objective function. However, the dynamic response is strongly dependent on the physical characteristics of the structure and thus the use of a deterministic set may lead to unexpected dynamic behaviors during different stages of design optimization.

To alleviate some of this issues, constraints such as frequency range, displacement and stresses were sometimes imposed in the optimization algorithms to tailor the structures dynamic response [1-3]; other approach was to specify a certain amount of material to attain a specific natural frequency [4]. These approaches relay heavily on a deterministic set of dynamic responses and could often lead to conservative and even off-optimal-performance designs.

Alternatively, several researchers [5-7] considered the inclusion of the dynamic behavior within the optimization problem through equivalent static load (ESL) sets that were computed using the solution of the transient analysis or approximated using modal analysis.

In the same spirit as [5-7], the dynamic behavior of any multi-degree of freedom system can be approximated through static modal participation factors (SMPF) as shown in [8]. The SMPF are inexpensive to compute and allow to assess the importance of a mode in the solution. Changes in modes with large participation factors, will then, induce large changes in the dynamic response. Thus, a response tracking mechanism based on static modal participation factors could lead to large computational savings and improve the optimization process by monitoring the dynamic response and the internal peak load of elements.

In this paper, an improved method based on SMPF is presented to approximate the modal peak responses when the structure is subjected to a shock or pulse disturbances. A case study is presented as a proof of concept, where the approximated peak out of plane bending moment at the wing root of an aircraft is approximated using SMPF.

\section{MODAL CONTRIBTION FACTORS}

The complex response of a dynamic structural system can be deconstructed into a set of simple harmonic motions $\mathrm{q}_{\mathrm{i}}(\mathrm{t})$, also known as modal responses, linearly coupled with amplitude ratios. This transformation is achieved by finding the system modal pairs in the form of natural frequencies $\omega_{i}$ and mode shapes $\Phi_{i}$ that satisfy the characteristic equation of undamped free vibration system which are employed to find the system response as the following variable change:

$$
\mathrm{u}(\mathrm{t})=\Phi \mathrm{q}(\mathrm{t})
$$

Where $u(t)$ is the nodal vector of physical displacements, and $\Phi$ is the modal matrix of column concatenated eigenvectors.

Expanding (1) in a summation form we obtain:

$$
\mathrm{u}_{\mathrm{n}}(\mathrm{t})=\phi_{\mathrm{n}, 1} \mathrm{q}_{1}(\mathrm{t})+\ldots+\phi_{\mathrm{n}, \mathrm{i}} \mathrm{q}_{\mathrm{i}}(\mathrm{t})
$$

From (2), the modal contribution can be defined as the quantity of movement that each mode contributes to the total nodal response $[9,10]$. In other words, it is the product between 
the modal response $\mathrm{q}_{\mathrm{i}}(\mathrm{t})$ and the amplitude ratio $\phi_{\mathrm{n}, \mathrm{i}}$, denoted here with the Greek letter gamma $(\gamma)$ as:

$$
\gamma_{n, i}(t)=\phi_{n, i} q_{i}(t)
$$

The amplitude of each modal response can be expressed as the product of a constant called modal participation factor $\Gamma_{\mathrm{i}}$ and a time dependent term $\mathrm{D}_{\mathrm{i}}(\mathrm{t})$ [9-11] which is expressed as:

$$
\mathrm{q}_{\mathrm{i}}(\mathrm{t})=\Gamma_{\mathrm{i}} \mathrm{D}_{\mathrm{i}}(\mathrm{t})
$$

Though in general the time history of the response may be of interest, it is the value of the maximum displacement that will be used for the sizing or the design optimization of a structure. Thus, from (5), the maximum value of the response is directly proportional to the maximum dynamic response which is expressed as:

$$
\mathrm{u}^{0}=\Phi \Gamma \mathrm{D}^{0}
$$

Where $\mathrm{u}^{0}$ is the vector of maximum nodal displacements, $\mathrm{D}^{0}$ is the dynamic modal response vector subjected to a unitary load and $\Gamma$ is the diagonal matrix of modal participation factors.

Appropriate expressions for $\Gamma$ and $\mathrm{D}^{0}$ can be found through static modal decomposition as addressed in the next section.

\section{STATIC MODAL PARTICIPATION FACTORS}

The distribution of external forces acting on the structure can be expressed as a time invariant vector $f$ and a time dependent expression $\mathrm{p}(\mathrm{t})$.

$$
\mathrm{F}(\mathrm{t})=f \mathrm{p}(\mathrm{t})
$$

Since the vector $f$ is independent of time we can try to estimate a static modal deflection caused by the distribution of the forces by expanding $f$ into a matrix of static forces $\beta^{3 t}$ acting at each node $j$ and within each mode $i$; where the sum of the columns of $f^{\text {st }}$ is equal to the magnitude of the $j$-th component of the vector $f$ [8-11] which is expressed as:

$$
f_{\mathrm{j}, \mathrm{i}}^{\mathrm{st}}=\Gamma_{\mathrm{i}, \mathrm{i}} \phi_{\mathrm{j}, \mathrm{i}} \quad\left\{\begin{array}{l}
\text { for } \mathrm{i}=1,2, \ldots, \mathrm{N} \\
\text { for } \mathrm{j}=1,2, \ldots, \mathrm{n}
\end{array}\right.
$$

Where $\mathrm{n}$ and $\mathrm{N}$ are the number of nodes and number of modes retained in the solution, respectively

$$
\Gamma=\operatorname{diag}\left(\Phi^{\mathrm{T}} \tilde{f}\right)
$$

When the eigenvectors are normalized with respect to mass, the vector of total static displacement can be found by the use of Hooke's law, which yields to:

$$
\mathrm{u}_{\mathrm{j}}^{\mathrm{st}}=\sum_{\mathrm{i}=1}^{\mathrm{N}} \frac{\Gamma_{\mathrm{i}, \mathrm{i}}}{\omega_{\mathrm{i}}^{2}} \phi_{\mathrm{j}, \mathrm{i}} \quad \text { for } \mathrm{i}=1,2, \ldots, \mathrm{N}
$$

The vector of total static displacement can then be related to the vector of total dynamic displacements by introducing (9) into (5) which results in:

$$
u_{j}^{o}=u_{j}^{s t} \omega_{i}^{2} D_{i}^{o}
$$

To derive an appropriate expression for $\mathrm{D}^{0}$, we begin by recalling the classical equation of motion in the modal coordinates, where the eigenvectors have been massnormalized:

$$
\ddot{\mathrm{q}}(\mathrm{t})+\omega_{\mathrm{i}}^{2} \mathrm{q}(\mathrm{t})=\Phi^{\mathrm{T}} f \mathrm{p}(\mathrm{t})
$$

Where the operator (*) denotes the second derivative with respect to time.

By introducing (4) and (8) into (11), we obtain (12), where the term $\Gamma$ is factored out of the resulting expression resulting in:

$$
\ddot{D}(\mathrm{t})+\omega_{i}^{2} D(t)=p(t)
$$

Equation (12) is simply the modal equations of motion subjected to a unit dynamic load; thus, depending on the form of the excitation force $\mathrm{p}(\mathrm{t})$, a particular method can be selected to find an analytical solution to (12) and consequently $\mathrm{D}^{0}$.

\section{A. Steady-state Response}

When $p(t)$ is a periodic function, a dynamic amplification factor $R_{i}$ can be defined as the ratio between the amplitudes of the dynamic response $\mathrm{D}_{i}^{o}$ and the static response $\mathrm{D}_{i}^{\text {st }}$ [9] expressed as:

$$
\mathrm{R}_{\mathrm{i}}=\frac{\mathrm{D}_{\mathrm{i}}^{\mathrm{o}}}{\mathrm{D}_{\mathrm{i}}^{\mathrm{st}}}
$$

The amplitude of the static response is found by neglecting the contribution of the acceleration term in the well-known mass-normalized modal equations of motion, yielding to (14).

$$
\mathrm{D}_{\mathrm{i}}^{\mathrm{st}}=\frac{1}{\omega_{\mathrm{i}}^{2}}
$$

The dynamic amplification factor is the steady-state amplitude of an undamped system subjected to a unit periodic function which is expressed as:

$$
\mathrm{R}_{\mathrm{i}}=\frac{1}{\left(\omega_{\mathrm{i}}^{2}-\omega^{2}\right)}
$$

By introducing (13-15) into (10), we find the inexpensive expression, i.e. (16) that allows to estimate the maximum amplitude of the nodal physical displacements during steadystate vibration, which is expressed as:

$$
u^{0}=u^{\text {st }} R
$$

To compare the output of this method with respect to the proposed methodology, a steady-state modal participation fraction $\mathrm{L}_{\mathrm{i}}^{\mathrm{st}}$ is defined in (17). This fractional number allows to compare the relative amplitudes of the modal responses $\mathrm{q}_{\mathrm{i}}$ for a given design and loading condition.

$$
\mathrm{L}_{\mathrm{i}}^{\mathrm{st}}=\frac{\left|\Gamma_{\mathrm{i}} \mathrm{D}_{\mathrm{i}}^{\mathrm{o}}\right|}{\sum_{\mathrm{i}}^{\mathrm{N}}\left|\Gamma_{\mathrm{i}} \mathrm{D}_{\mathrm{i}}^{\mathrm{o}}\right|}=\frac{\left|\Gamma_{\mathrm{i}} /\left(\omega_{\mathrm{i}}^{2}\left(\omega_{\mathrm{i}}^{2}-\omega^{2}\right)\right)\right|}{\sum_{\mathrm{i}}^{\mathrm{N}}|| \Gamma_{\mathrm{i}} /\left(\omega_{\mathrm{i}}^{2}\left(\omega_{\mathrm{i}}^{2}-\omega^{2}\right)\right) \mid}
$$

\section{B. Transient Response I}

The steady participation factors neglect the contribution of the transient components of the time domain solution. When the global maximum is of particular interest, we aim here to estimate the maximum dynamic amplitude of system response computed as:

$$
\mathrm{D}_{\mathrm{i}}^{\max }=\max (\mathrm{D}(\mathrm{t}))
$$

The transient participation fraction $L_{i}^{t s}$ can then be defined similarly to the steady participation fraction as the ratio between the maximum amplitude of the harmonic solution and the sum of the maximum amplitude of all the modes retained in the system which is expressed as: 


$$
\mathrm{L}_{\mathrm{i}}^{\mathrm{ts}}=\frac{\left|\Gamma_{\mathrm{i}} \mathrm{D}_{\mathrm{i}}^{\max }\right|}{\sum_{\mathrm{i}}^{\mathrm{N}}\left|\Gamma_{\mathrm{i}} \mathrm{D}_{\mathrm{i}}^{\max }\right|}
$$

\section{Transient Response II - Analytical solutions}

To obtain (19) the solution of the modal equations of motion, necessary for (18) calculations, is required a priori. As shown in [12] the solution of (18) was approximated using a quasi-static solution. Here we introduce a new participation factor based on the closed form solution of a Single Degree of Freedom System (SDOF).

Since this method requires the analytical solution of the modal equations of motion in the time domain, we use here the definition given in (20) as an example of an excitation signal which represent the analytical model of Tuned Discreet Gust (TDG) of an aero structure. However the methodology is applicable for any forcing function where a closed form solution exists.

$$
p(t)=1-\cos (\omega t)
$$

where $\omega$ is the forcing frequency.

The analytical solution in the time domain for an undamped SDOF system subjected to a unitary pulse, as shown in (20), is given by:

$$
D_{i}=\left\{\begin{array}{cr}
\frac{1}{\omega_{\mathrm{i}}^{2}}\left(1-\cos \left(\omega_{\mathrm{i}} \mathrm{t}\right)\right)-\frac{2}{\omega^{2}-\omega_{\mathrm{i}}^{2}} \sin \left(\frac{\left(\omega_{\mathrm{i}}+\omega\right) \mathrm{t}}{2}\right) \sin \left(\frac{\left(\omega_{\mathrm{i}}-\omega\right) \mathrm{t}}{2}\right) & 0 \leq \mathrm{t} \leq \tau \\
\frac{1}{\omega_{\mathrm{i}}^{2}}\left[\cos \left(\omega_{\mathrm{i}}(\mathrm{t}-\tau)\right)-\cos \left(\omega_{\mathrm{i}} \mathrm{t}\right)\right]+\frac{1}{2 \omega_{\mathrm{i}}\left(\omega-\omega_{\mathrm{i}}\right)}\left[\cos \left(\left(\omega-\omega_{\mathrm{i}}\right) \tau+\omega_{\mathrm{i}} \mathrm{t}\right)-\cos \left(\omega_{\mathrm{i}} \mathrm{t}\right)\right]- \\
\cdots \frac{1}{2 \omega_{\mathrm{i}}\left(\omega+\omega_{\mathrm{i}}\right)}\left[\cos \left(\left(\omega+\omega_{\mathrm{i}}\right) \tau-\omega_{\mathrm{i}} \mathrm{t}\right)-\cos \left(\omega_{\mathrm{i}} \mathrm{t}\right)\right] & \tau \leq \mathrm{t}
\end{array}\right.
$$

Where $\tau$ is the pulse length. From the classical theory of shock, the maximum response will occur during the application of the pulse whenever the forcing frequency is smaller than twice the natural frequency of the system [13]. Thus, the solution for the maximum amplitude of the dominant elastic mode and the time $t_{1}^{\max }$ at which it occurs can be estimated by differentiating (21) with respect to time during the application of the pulse and finding the roots of the derivative; alternatively, computing the complete time response of the dominant mode using (21) and extracting the peak values.

Then, the rest of the dynamic responses can be estimated by computing (20) at $t_{1}^{\max }$, that is:

$$
D_{i}^{\max }=D_{i}\left(t_{1}^{\max }\right)
$$

The transient response II participation fraction is defined similar to equations (17) and (19) as:

$$
\mathrm{L}_{\mathrm{i}}^{\mathrm{tsII}}=\frac{\left|\Gamma_{\mathrm{i}} \mathrm{D}_{\mathrm{i}}^{\max }\right|}{\sum_{\mathrm{i}}^{\mathrm{N}}\left|\Gamma_{\mathrm{i}} \mathrm{D}_{\mathrm{i}}^{\max }\right|}
$$

\section{Internal load Participation Factor}

For point loads, the element internal forces are computed as the product of the element stiffness matrix $\mathrm{K}_{\mathrm{e}}$ and the corresponding displacement vector $\mathrm{U}_{\mathrm{e}}(\mathrm{t})$ of the nodes that conform the element in the global coordinate system.

When the element and global coordinate systems do not coincide a rotation matrix $\mathrm{C}^{\mathrm{T}}$ is necessary to ensure that the internal forces computed, $\mathrm{N}_{\mathrm{e}}(\mathrm{t})$, are expressed in the element coordinate system as:

$$
\mathrm{N}_{\mathrm{e}}(\mathrm{t})=\mathrm{C}^{\mathrm{T}} \mathrm{K}_{\mathrm{e}} \mathrm{U}_{\mathrm{e}}(\mathrm{t})
$$

Where $\mathrm{K}_{\mathrm{e}}$ and $\mathrm{U}_{\mathrm{e}}(\mathrm{t})$ are the element stiffness matrix and the vector of nodal displacements in the global coordinate system., respectively.

We can expand equation (24) into its modal components as:

$$
\mathrm{N}_{\mathrm{e}}(\mathrm{t})=\mathrm{C}^{\mathrm{T}} \mathrm{K}_{\mathrm{e}} \Phi_{\mathrm{e}} \mathrm{q}(\mathrm{t})=\mathrm{C}^{\mathrm{T}} \mathrm{K}_{\mathrm{e}} \Phi_{\mathrm{e}} \Gamma \mathrm{D}(\mathrm{t})
$$

Equation (23) gives an important insight into the variables that affect the internal loads sustained by each element. The term $\Gamma$ is constant for a given loading conditions and its magnitude depends on the matrix of eigenvectors. On the other hand, the magnitude of $\mathrm{D}(\mathrm{t})$ is strongly dependent on the initial conditions and the ration between the forcing and the natural frequencies of the system. The magnitude of $\Gamma \mathrm{D}(\mathrm{t})$, thus, determines the dominance of a mode in the solution, whereas the product $\Sigma_{\mathrm{e}}=\mathrm{C}^{\mathrm{T}} \mathrm{K}_{\mathrm{e}} \Phi_{\mathrm{e}}$ determines the impact of such mode in the $\mathrm{r}$ component of the element load where $r \in\{1: 6\}$. A participation factor can then be defined as:

$$
\mathrm{L}_{\mathrm{r}, \mathrm{i}}^{\text {load }}=\frac{\mid \Sigma_{\mathrm{e}_{\mathrm{r}, \mathrm{i}} \Gamma_{\mathrm{i}} \mathrm{D}_{\mathrm{i}} \mid}}{\sum_{\mathrm{i}=1}^{\mathrm{N}}\left|\Sigma_{\mathrm{e}_{\mathrm{r}, \mathrm{i}}} \Gamma_{\mathrm{i}} \mathrm{D}_{\mathrm{i}}\right|}
$$

\section{CASE STUDY}

An aircraft stick model based on a Bombardier Aircraft platform was created to assess the performance of each of the methods described herein. The model is excited by a forcing vector $\mathrm{f}$ with a linear distribution of a $1000 \mathrm{~N}$ on the wing root and $480 \mathrm{~N}$ on the wing tip of the form given in equation (20). The modal responses were calculated in MSC Nastran [14] and the normalized results are displayed in Figure 2.

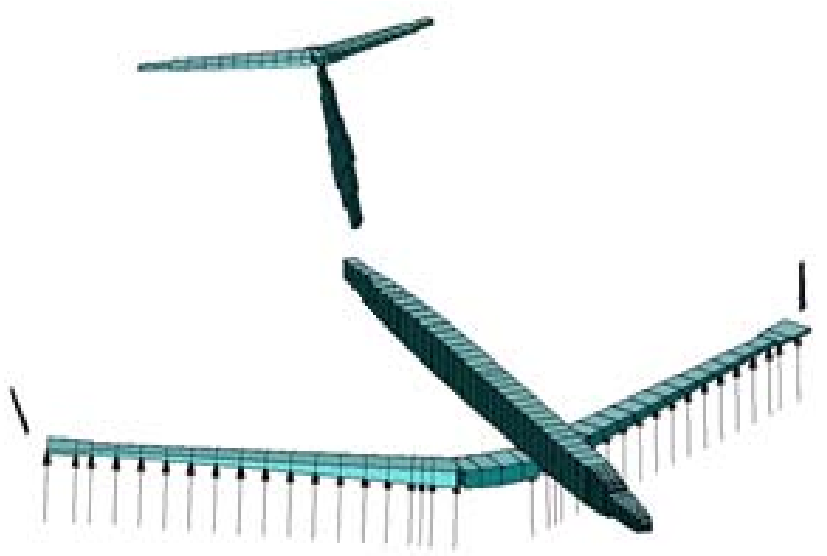

Figure 1. Case study: airplane stick model. 


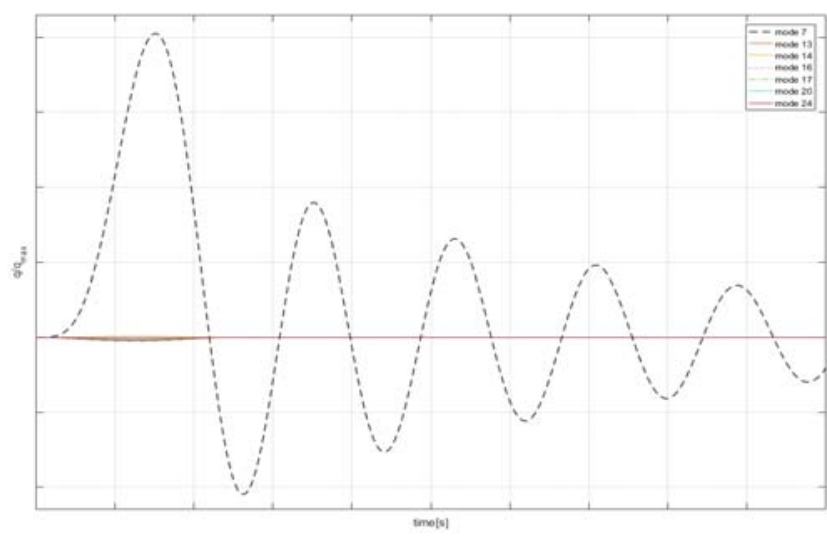

Figure 2. Normalized modal responses.

The modal participation factors of the free-free airplane model were computed using (17), (19) and (23) and the results are presented in Table I.

The results are well in agreement with the transient response of Figure 1; the first elastic mode dominates entirely the response, followed by the elastic modes 7,8 and 10 . The peak out of plane bending moment of the wing root was estimated also in MSC Nastran, and approximated using (25) with (17), (19) and (23). The percentage of error is shown in figure 3.

TABLE I. MODAL PARTICIPATION FACTORS

\begin{tabular}{|c|c|c|c|}
\hline \multicolumn{4}{|c|}{ Modal Participation Fractions } \\
\hline Elastic mode & $L_{i}^{t s}$ & $\mathrm{~L}_{\mathrm{i}}^{\text {tsII }}$ & $\mathrm{L}_{\mathrm{i}}^{\text {st }}$ \\
\hline 1 & $94.67 \%$ & $95.41 \%$ & $95.93 \%$ \\
\hline 2 & $0.00 \%$ & $0.00 \%$ & $0.00 \%$ \\
\hline 3 & $0.00 \%$ & $0.00 \%$ & $0.00 \%$ \\
\hline 4 & $0.00 \%$ & $0.00 \%$ & $0.00 \%$ \\
\hline 5 & $0.00 \%$ & $0.00 \%$ & $0.00 \%$ \\
\hline 6 & $0.00 \%$ & $0.00 \%$ & $0.00 \%$ \\
\hline 7 & $0.21 \%$ & $0.18 \%$ & $0.16 \%$ \\
\hline 8 & $0.27 \%$ & $0.22 \%$ & $0.20 \%$ \\
\hline 9 & $0.00 \%$ & $0.00 \%$ & $0.00 \%$ \\
\hline 10 & $0.31 \%$ & $0.27 \%$ & $0.24 \%$ \\
\hline
\end{tabular}

Both of the transient approximations present better results than that of the steady-state participation factor, which has an error of $32 \%$. This result is expected since the modal amplitude computed using (17) treats the excitation as a periodic function.

That is, even though the participation factor obtained using $L_{i}^{\text {st }}$ is well in agreement with Figure 1, the actual magnitude of the modal response is not. This large variation is reflected in the large error of the estimated out of plane bending moment.

The static modal participation factor $L_{i}^{t s}$ was computed using the time domain solution of the modal response extracted from
Nastran; its associated error is due an implicit assumption of equation (18): the modal responses are considered to be in phase. This means that the recovered amplitudes are not time consistent, thus the method tends to either over or underestimate the value of the internal load, even when the exact solution is known.

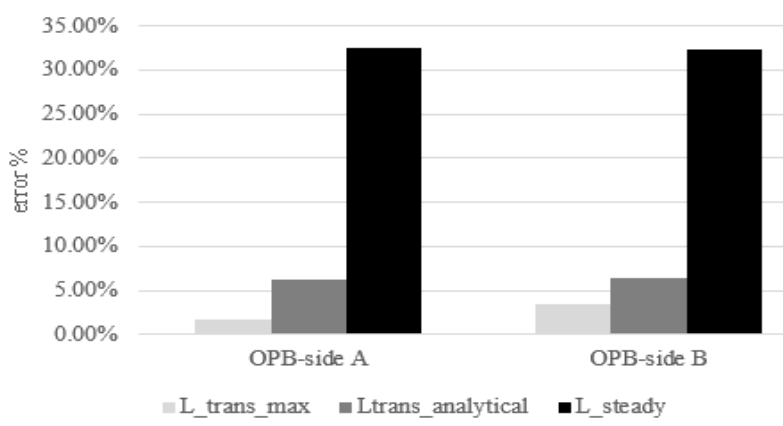

Figure 3. Normalized modal responses.

In the case of $\mathrm{L}_{\mathrm{i}}^{\text {tsII }}$ the approximation could be improve by considering modal damping in the derivation of the analytical solution (23).

Finally, the modal participation fraction of the out of plane bending moment at the wing root were computed using (26) with (17), (19) and (23). The results are shown and compared in Table II.

TABLE II. ELEMENT INTERNAL LOAD MODAL PARTICIPATION FACTORS

\begin{tabular}{|c|c|c|c|}
\hline \multicolumn{4}{|c|}{ Out of Plane Bending Moment at wing root contribution fractions } \\
\hline Elastic mode & $L_{i}^{t s}$ & $\mathrm{~L}_{\mathrm{i}}^{\mathrm{tsI}}$ & $\mathrm{L}_{\mathrm{i}}^{\text {st }}$ \\
\hline 1 & $92.449 \%$ & $93.444 \%$ & $94.263 \%$ \\
\hline 2 & $0.000 \%$ & $0.000 \%$ & $0.000 \%$ \\
\hline 3 & $0.000 \%$ & $0.000 \%$ & $0.000 \%$ \\
\hline 4 & $0.000 \%$ & $0.000 \%$ & $0.000 \%$ \\
\hline 5 & $0.000 \%$ & $0.000 \%$ & $0.000 \%$ \\
\hline 6 & $0.000 \%$ & $0.000 \%$ & $0.000 \%$ \\
\hline 7 & $0.196 \%$ & $0.168 \%$ & $0.152 \%$ \\
\hline 8 & $0.195 \%$ & $0.159 \%$ & $0.150 \%$ \\
\hline 9 & $0.000 \%$ & $0.000 \%$ & $0.000 \%$ \\
\hline 10 & $0.056 \%$ & $0.049 \%$ & $0.043 \%$ \\
\hline
\end{tabular}

Once again, the first elastic mode dominates entirely the response. However, it is noticed that the participation of the modes shown in Table II decrease. This means that term $\Sigma_{\mathrm{e}}$ from equation (25) increased the participation of higher order modes.

\section{CONCLUSIONS AND FUTURE WORK}

Static modal participation factors have the potential to be used as response tracking mechanisms during structural design optimization, since they are inexpensive to compute and allow 
to estimate the peak internal load of the elements. Thus, large design spaces could be easily updated without the need to run any dynamic analysis.

The methodology presented herein is applicable for systems with concentrated nodal forces, however, in aerospace applications we mostly encounter distributed loads such as aerodynamic and inertia loads. The extension of this methodology for aeroelastic response analysis is of particular interest and will be addressed in our future work.

\section{REFERENCES}

[1] Grandhi, R. (1993). Structural Optimization with Frequency Constraints: A Review, 31(12), 2296-2303.

[2] Rao, S. S. (1985). Optimization of airplane wing structures under gust loads. Computers and Structures, 21(4), 741-749.

[3] Rao, V. R., Iyengar, N. G. R., \& Rao, S. S. (1979). Optimization of wing structures to satisfy strength and frequency requirements. Computers and Structures, 10(4), 669-674.

[4] Vanderplaats, G. N., \& Problem, T. D. (1981). Structural OptimizationPast, Present, and Future. AIAA journal, 20(7), 992-1000.

[5] Choi, W. S., \& Park, G. J. (1999). Transformation of dynamic loads into equivalent static loads based on modal analysis. International Journal for Numerical Methods in Engineering, 46(1), 29-43.
[6] Choi, W. S., \& Park, G. J. (2002). Structural optimization using equivalent static loads at all time intervals. Computer Methods in Applied Mechanics and Engineering, 191(19-20), 2105-2122.

[7] Choi, W. S., Park, K. B., \& Park, G. J. (2005). Calculation of equivalent static loads and its application. Nuclear engineering and design, 235(22), 2337-2348.

[8] Romera, L. E., \& Hernandez, S. (1999). An improved technique for modal contribution factors of dynamic responses. In AIAA/ASME/ASCE/AHS/ASC Structures, Structural Dynamics, and Materials Conference and Exhibit, 40 th, St. Louis, MO (pp. 439-445).

[9] Chopra, A. K. (1996). Modal analysis of linear dynamic systems: physical interpretation. Journal of structural engineering, 122(5), 517-527.

[10] Chopra, A. K. (2001). Dynamics of structures: Theory and applications. Prentice Hall.

[11] Palermo, M., Silvestri, S., Gasparini, G., \& Trombetti, T. (2015). Seismic modal contribution factors. Bulletin of Earthquake Engineering, 13(10), 2867-2891.

[12] Wallrapp, O., \& Wiedemann, S. (2002). Simulation of deployment of a flexible solar array. Multibody System Dynamics, 7(1), 101-125.\{13] Press. De Silva, C. W. (Ed.). (2005). Vibration and shock handbook. CRC

[13] De Silva, C. W. (Ed.). (2005). Vibration and shock handbook. CRC Press.

[14] MacNeal, R. H. (Ed.). (1970). The NASTRAN theoretical manual (Vol. 221). Scientific and Technical Information Office, National Aeronautics and Space Administration. 